\title{
The development of shared cognition in paediatric residents analysing a patient video versus a paper patient case
}

\author{
T. Balslev • W. de Grave • A. M. M. Muijtjens • B. Eika • \\ A. J. J. A. Scherpbier
}

Received: 28 January 2008/ Accepted: 22 September 2008

(C) Springer Science+Business Media B.V. 2008

\begin{abstract}
In a previous study, we established that compared to a written case, a video case enhances observable cognitive processes in the verbal interaction in a postgraduate problem-based learning format. In a new study we examined non-observable cognitive processes using a stimulated recall procedure alongside a reanalysis of the data from the first study. We examined the development of shared cognition as reflected in collaborative concept link formation, an approach to connecting a series of concepts related to a particular topic. Eleven paediatric residents were randomly allocated to two groups. After both analysing the same written case vignette, one group watched a video of the case in the vignette and the other group read a written description of the video. Both groups then reanalysed the vignette. After the group sessions, time-logged transcripts were made of the verbal interaction in both groups and all residents individually took part in a stimulated recall procedure. Causal reasoning concept links were labelled as individual or collaborative depending on whether they originated from individual residents or were directly elicited by verbal utterances from others. The video led to a significantly increased frequency ratio (after intervention: before intervention) of collaborative concept links but did not affect the frequency of individual concept links. This novel process approach to chronological registration of concept link formation offered additional evidence that shared
\end{abstract}

T. Balslev

Department of Paediatrics, Aarhus University Hospital, Skejby, Denmark

T. Balslev ( $\bowtie)$

Gl Skørpingvej 114, 9520 Skorping, Denmark

e-mail: bafl@dadlnet.dk

W. de Grave · A. M. M. Muijtjens

Department of Educational Development and Research, Maastricht University,

Maastricht, The Netherlands

B. Eika

Unit of Medical Education, Aarhus University, Aarhus, Denmark

A. J. J. A. Scherpbier

Institute for Medical Education, Faculty of Health, Medicine and Life Sciences, Maastricht University, Maastricht, The Netherlands 
cognition by means of co-elaboration of concept formation is stimulated by the use of patient video recordings in small group learning.

Keywords Collaborative learning - Paediatric resident education - Patient video case . Postgraduate training $\cdot$ Resident education $\cdot$ Shared cognition $\cdot$ Video

\section{Introduction}

Diagnosing clinical cases is not always a straightforward task for residents in paediatric training. Residents can improve their clinical reasoning skills by tackling clinical cases where the diagnosis is not given, because such cases mimic the reality of clinical practice (Eva 2005). It also appears desirable to supplement the number of real patients that residents can see during their training with extra cases, since expertise is largely built through the matching of problems against previously encountered similar problems. Additional cases will enable residents to generate and store in memory an increased number of instance scripts (Schmidt et al. 1990). The extra cases may take the form of patient video cases (PVC), which can present characteristic signs and symptoms, are highly authentic and easy to obtain.

Watching PVCs and discussing them with peers may give learners challenges they might not otherwise have (Westberg and Jason 1994). When learners interact and exchange ideas in a group, cognitive processes are stimulated. In one of these processes, that of co-elaboration, knowledge is generated when participants in a discussion extend each other's ideas (Slavin 1996; Dolmans 1998; Visschers-Pleijers et al. 2006). It can be helpful to learning when learners discern links between a series of concepts related to a particular topic, so-called concept link formation (Novak and Gowin 1984).

Shared cognition is an essential feature of collaborative learning from clinical encounters (Lebeau 1998). It extends beyond shared knowledge because it also includes the processes and products of clinical reasoning and builds upon individuals' inputs in the collaborative process. Collaborating individuals share cognition when they meaningfully integrate new knowledge into their cognitive structure using a similar frame of reference to interpret this knowledge. (Bossche and den Gijselaers 2006)

There is a paucity of instruments to measure change in individuals' cognitive structures during group interaction (Mohammed et al. 2000). Hence, we know little about the cognitive processes that occur when learners collaboratively analyse video or paper patient cases. Our previous study of verbal interaction in a group of paediatric residents showed that a PVC induced cognitive processes that were superior to those triggered by paper cases (Balslev et al. 2005). However, the analysis of the cognitive processes in this study was restricted to the recorded verbal interactions in the group.

As the observable verbal interactions alone does not yield enough information to gain in-depth insight into the cognitive processes of shared cognition, we also explored the residents' unexpressed thoughts generated during the group interaction (Barrows 2000). During verbal interaction in a group only one person is speaking at a time and thus it is only the thoughts of one person that are being voiced at that moment, although presumably the others will be thinking as well. This implies that only the tip of the iceberg of participants' individual thought processes is revealed during verbal group interaction.

We expected that concept links would evolve over time depending on collaboration among the residents. In our previous study, we focused on concept links related to causal clinical reasoning. We were specifically interested in the interaction between individual 
and group as evidenced by the frequency of concept link formation in individual residents as a result of the verbal interaction in the group. The group's shared goal of diagnosing an authentic patient was assumed to be a strong stimulus for co-elaboration by means of collaborative concept link formation, particularly in the group who watched the PVC. In the current paper, we present a reanalysis of the data from the verbal interaction alongside new data from a stimulated recall procedure to explore if a PVC not only generates increased verbal interaction but also enhances shared cognition.

We formulated the following research question: Will supplementing a written case vignette by a PVC as opposed to an equivalent paper case increase shared cognition as measured by the frequency of collaborative concept link formation in the context of collaborative learning about movement disorders in the first three years of paediatric residency?

\section{Methods}

Participants and groups

Sixteen eligible residents who had completed up to 36 months of paediatric training (mean 21 months) in the same region in Denmark were invited to participate, and 12 residents agreed to participate. The residents were familiar with the format and were comfortable with video recording after their participation in a series of pilot studies with paediatric video cases. The first author acted as tutor in the group sessions. Clusters of matching residents composed on the basis of gender and months of paediatric training were formed. Of each cluster $50 \%$ of the residents were randomly allocated to Group A, and the remaining ones to Group B. One resident did not participate due to illness. Group A consisted of five residents and group B of six residents. A flow-chart (Fig. 1) summarizes the study process.

\section{The case}

The case involved a 2-month-old male infant with Sturge-Weber syndrome, a rare congenital disease of which a facial haemangioma is a common symptom. The video showed an infant with a haemangioma on the forehead and an ongoing partial motor seizure on the right side of the body. The problem was chosen because most paediatric residents can be expected to be familiar with some of the signs and symptoms while the problem is sufficiently challenging to trigger cognitive processes.

\section{Group sessions}

Both groups analysed the same written vignette of the case and formulated learning objectives after the analysis (Balslev et al. 2005). Next, group B was shown a brief ( $2.5 \mathrm{~min}$ ) video of the patient in the vignette, whilst group A read a written description of the video without actually watching it. The written description listed what could be seen in the video without any diagnostic hints. For instance, in the text the terms "jerking" in stead of "seizure" and "blue spot" in stead of "haemangioma"; were used. The groups then repeated the analysis. While analysing the case group B could watch the video and group A could read the text more than once. Group A initially read the text silently. Group B 


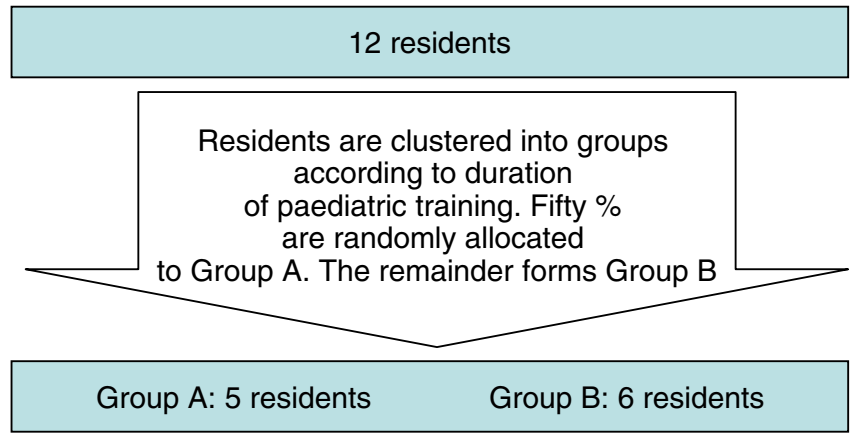

Group A (text)

Residents read the written vignette, analyze and interact
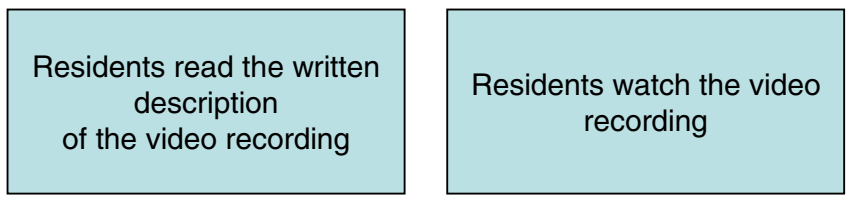

Residents analyze and interact

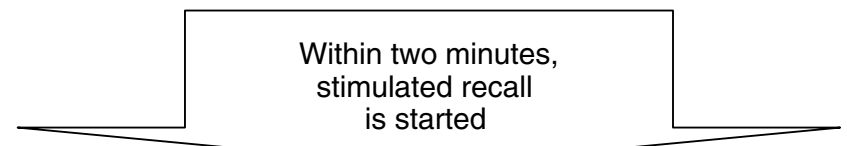

\section{Individual, stimulated recall}

The video of the interaction is replayed for each resident. An interviewer stimulates the resident to verbalise his/hers thinking processes
One resident went ill and did not participate
Interaction is recorded on video
The stimulated recall is recorded on audiotape

Two time-logged transcripts of the two videorecordings and 5+6 audiotapes with stimulated recall are made. Causal reasoning concept links are identified

Fig. 1 This flow diagram summarizes the one-day study process

watched the video twice. More details on study design and methods have been published elsewhere (Balslev et al. 2005).

Stimulated recall

Since it is not possible to register individual residents' thinking processes unobtrusively while the discussion is ongoing, we used stimulated recall as a means to tap into these processes. 
The stimulated recall procedure involved one-on-one contact of a resident and an interviewer. The interviewers were not blinded. Two of the authors, TB and BE, and four paediatric specialists conducted the interviews. The interviewer introduced him/ herself and explained the stimulated recall procedure to the resident. Within two minutes, the video of the group session was played to the resident to trigger recall of the discussion. The residents were invited to recount all that they remembered of what they had been thinking during the discussion and the video was stopped every time a resident remembered something. It was the interviewer's task to stimulate the residents to verbalise their thinking processes. After the actual recall procedure, brief individual interviews were conducted to validate the stimulated recall. The residents from group A were asked "Please describe what it was like to analyse a case from a printed text" and the residents from group B were asked the same question but about a PVC.

\section{Data collection and analysis}

Interaction in the groups was recorded on video and transcribed verbatim. Our aim was to capture concept link formation over time as clinical reasoning processes were ongoing in the group and in individual residents. We assumed that concept link formation was highly dependent on collaboration in the group and would emerge chronologically in the timelogged transcription of the verbal interaction in the group and in the stimulated recall. As concept mapping is not adequate for measuring the sharing of concepts, (Mohammed et al. 2000) we used verbal protocol analysis (Ericsson and Simon 1993) to better capture the cognitive processes. Time-logged transcripts were produced with columns for verbal group interaction and individual stimulated recall. Two raters independently identified causal reasoning concept links in both the verbal interaction and the stimulated recall. To map the processes of concept link formation, we identified the concept links as they occurred chronologically.

We distinguished two types of causal reasoning concept links (Table 1), both of them easily recognizable in the time logged transcripts. The first type was the collaborative concept link, directly elicited by verbal utterances of other participants, i.e. following within a few seconds, and linked to it in content. One utterance by one of the residents could prompt a number of collaborative concept links in one or more residents at the same time. These could be verbalized, or more frequently, not verbalized. The second type was the individual causal reasoning concept link, produced by individual residents. An example was an utterance by a residence followed by an individual concept link in that particular resident only and not in the other residents. The inter-rater reliability of this categorisation was determined by generalisability analysis and calculation of intra-class correlation coefficients. The two raters analysed four representative data sets, each consisting of six two-minute samples of the discussions in groups A and B before and after reading the text and watching the video, respectively. With two raters, Generalisability $=\operatorname{Var}($ sample $) /[\operatorname{Var}($ sample $)+\operatorname{Var}($ rater $\times$ sample $) /$ Nrater $]$ was 0.76 for individual links and 0.94 for collaborative links. Thus two raters were sufficient for the final analysis of our data.

We calculated the mean frequencies of the two types of concept links. Chi-square tests were used to determine the significance of any differences between Groups A and B after and before the intervention. 
Table 1 Differences between Group A (text) and Group B (video) in the number of collaborative and individual concept links

\begin{tabular}{lcccll}
\hline Categories & $\begin{array}{l}\text { Before } \\
\text { intervention }\end{array}$ & $\begin{array}{l}\text { After } \\
\text { intervention }\end{array}$ & Total & $\begin{array}{l}\text { Ratio after/before } \\
\text { intervention }\end{array}$ & $\begin{array}{l}\text { Difference } \\
\text { A and B }\end{array}$ \\
\hline $\begin{array}{l}\text { Collaborative concept links } \\
\text { A (text) }\end{array}$ & 60 & 61 & 121 & 1.0 & Chi-square $=7.20$ \\
B (video) & 73 & 138 & 211 & 1.9 & $\begin{array}{l}\text { d.f. }=1 \\
P\end{array}$ \\
Total & 133 & 199 & 332 & & 0.01 \\
Individual concept links & & & & \\
A (text) & 22 & 31 & 53 & 1.4 & $\begin{array}{l}\text { Chi-square }=0.02 \\
\text { d.f. }=1\end{array}$ \\
B (video) & 30 & 40 & 70 & 1.3 & N.S. \\
Total & 52 & 71 & 123 & & \\
\hline
\end{tabular}

The number of concept links and the after/before intervention ratios of the numbers of links are shown

a The final column shows the results of the Chi-square analysis of the differences between Group A and Group B; d. f. = degrees of freedom

\section{Results}

A total of 455 causal reasoning concept links were identified, $112(25 \%)$ in the verbal interaction and $343(75 \%)$ during the stimulated recall procedure. This appears to confirm the importance of stimulated recall for in-depth analysis of cognitive processes.

The video group showed a significant increase in collaborative concept links but not in individual concept links after watching the video (Table 1).

In Table 2, sample clauses from the stimulated recall are shown to give an impression of the intense co-elaboration of visual representations (pictorial models) when the group was viewing the video. The shared cognitive processes as assessed by collaborative concept link formation were more intense after the group had watched the video for the second time.

Many residents in group B commented the video while watching it in contrast to the silence observed during the initial reading of the residents of the text group. Individual as

Table 2 Sample clauses from the verbal interaction and the stimulated recall after small group analysis of a Sturge Weber case, illustrating co-elaboration of the pictorial model during the first viewing of the patient video

\begin{tabular}{|c|c|}
\hline Verbal interaction & Stimulated recall \\
\hline A: He has a convulsion & B: Immediately, I see the haemangioma \\
\hline $\begin{array}{l}\mathrm{C} \text { : There is something in the } \\
\text { right foot }\end{array}$ & B: When I see the jerking I think epileptic seizure \\
\hline D: The arm too, yes... & D: I see the fine jerking of the foot \\
\hline E: I see it... & E: We are looking at a child in a serious condition: he is not sleeping! \\
\hline $\mathrm{C}$ : This is clonic... & $\begin{array}{l}\text { C: I see the little tics of the foot. That is the first thing I see. Later I see the } \\
\text { arm and eyes. I can make a good description of the seizure } \\
\text { F: I think the right arm is jerking, and I think of the spot on the forehead. I } \\
\text { think he has secretions or maybe he is hyperventilating }\end{array}$ \\
\hline B: He has & B: I observe him, trying to see what I can see concerning the seizure \\
\hline
\end{tabular}
eye 
well as collaborative concept links were noted during both of the reviews of the video. During the silent reading of the text in the text group, causal concept links were scarce or absent.

A small difference was observed in the duration of the problem analysis before and after the interventions: Group A (text): 20/23 min, Group B (video): 22/28 min.

In the validating interviews some of the residents who had analysed the text said a video recording would have been helpful in analysing the case or creating pictorial models. All the residents who had watched the PVC reported that it showed posture and/or movements better than a text would do.

\section{Discussion}

The results show that adding a PVC to a written vignette enhanced shared cognition through collaborative concept link formation among paediatric residents analysing a case in a small group. We used a novel process approach to chronological registration of concept link formation, which showed that co-elaboration of concept formation is stimulated when residents watch a PVC during small group learning. This information is new and in line with theory of collaborative learning (Dillenbourg 1999). It seems that, compared to a paper case, a video case is more effective in facilitating the sharing of cognitive processes by learners engaged in team learning.

The observation that many residents commented the video while watching it in contrast to the silence during the initial reading of the residents of the text group may have contributed to an earlier onset of both types of causal concept links formation. This is important to note as the use of video in stead of a text case may help learners to obtain an immediate effect on learning.

The results of this study suggest that the construction of similar pictorial models in the residents' working memories facilitated collaborative concept formation. This is consistent with cognitive theory of multimedia learning (Mayer 2001). It is worth mentioning that very intense 'brainstorming', leading to collaborative concept link formation was only seen after the group had watched the PVC for the second time. This delay suggests that watching and processing the PVC may have caused cognitive overload (Paas et al. 2003), thereby slowing down concept link formation. Very importantly, the intense collaborative concept link formation after the second viewing suggests that multiple replays of PVCs interspersed with discussion will be beneficial to learners engaged in collaborative analysis of a PVC. Perhaps an approach that involves repeated viewing of a PVC after group coelaboration can be regarded as a new addition to load-reducing methods for overload scenarios in multimedia instruction as listed by Mayer et al. (Mayer and Moreno 2003).

The finding of a higher inter-rater reliability for collaborative links may indicate that identification of individual links is more difficult than identification of collaborative links in a transcript.

The main strength of this study is that it affords additional insight into collaborative activation of concept links by the use of stimulated recall to tap into residents' thoughts that were not expressed during group discussion.

The fact that more time was spent analysing the case after watching the video than after reading the text suggests that these different media trigger different cognitive processes. The longer duration of the analysis after watching the video is consistent with findings from a previous controlled study in a paediatric clerkship where students spent more time interpreting a video than interpreting a corresponding text (Kamin et al. 2001). However, 
the present study consisted of a single experiment in a limited number of participants and more research will be needed to establish with greater certainty whether and why analysis of a PVC requires more time than that of an equivalent text.

Some shortcomings of the present study must be kept in mind. Firstly, this is a small study of 11 residents only, analysing a single case. Secondly, the frequency of collaborative concept links is only one facet of shared cognition among others that can also be taken as a measure of shared cognitive processes. The validity and reliability of stimulated recall have not yet been conclusively established. Researchers cannot be sure that all cognitive processes are recalled nor that all cognitive processes are verbalised during stimulated recall. However, the fact that the stimulated recall procedure took place immediately after the verbal interaction in the group and yielded $75 \%$ of the identified concept links, suggests that a substantial part of the concepts formed were indeed picked up. Thirdly, the interviewers were not blinded to the group origin of their residents, a potential bias to the study-design used. We believe, however, that the dependent variables were unlikely to be affected by suggestions of the interviewers. The interviewers were instructed to stimulate the residents to verbalize their thoughts, and not to stimulate verbalization of selective cognitive processes.

This study illuminates shared cognition among junior doctors analysing a video case. Day-to-day clinical practice, however, usually implies teams consisting of medical students, residents and specialists, with different clinical experience and backgrounds. Therefore, new research might focus on interactive approaches involving video cases supporting cognitive processes in a spectrum of learners as well as experienced clinicians, by simulating clinical work in clinical departments. Learning gains may be greater for some learners than for others (Clark and Craig 2001).

\section{References}

Balslev, T., de Grave, W. S., Muijtjens, A. M. M., \& Scherpbier, A. J. J. A. (2005). Comparison of text and video cases in a postgraduate pbl-format with respect to the cognitive and metacognitive processes induced. Medical Education, 39, 1086-1092. doi:10.1111/j.1365-2929.2005.02314.x.

Barrows, H. S. (2000). Stimulated recall (personalized assessment of clinical reasoning). Illinois: Southern Illinois University, School of Medicine.

Bossche, P. V., \& den Gijselaers, W. H. (2006). Social and cognitive factors driving teamwork in collaborative learning environments. Team learning beliefs and behaviours. Small Group Research, 37, 490-517. doi:10.1177/1046496406292938.

Clark, R. E., \& Craig, T. B. (2001). Learning from media. Arguments, analysis and evidence. In R. E. Clark (Ed.), What about multimedia effects on learning? (pp. 89-101). Greenwich, Connecticut: Information Age Publishing.

Dillenbourg, P. (1999). What do you mean by “collaborative learning”? In P. Dillenbourg (Ed.), Collaborative learning: Cognitive and computational approaches (pp. 1-19). Oxford: Elsevier.

Dolmans, D. H. J. M. (1998). Motivational and cognitive processes influencing tutorial groups. Academic Medicine, 73, 22-24. doi:10.1097/00001888-199810000-00034.

Ericsson, K., \& Simon, H. A. (1993). Protocol analysis: Verbal reports as data. Cambridge, MA: The MIT Press.

Eva, K. W. (2005). What every teacher needs to know about clinical reasoning. Medical Education, 39, 98-106. doi:10.1111/j.1365-2929.2004.01972.x.

Kamin, C. S., O’Sullivan, P. S., Younger, M., \& Derterding, R. (2001). Measuring critical thinking in problem-based learning discourse. Teaching and Learning in Medicine, 13, 27-35. doi:10.1207/ S15328015TLM1301_6.

Lebeau, R. B. (1998). Cognitive tools in a clinical encounter in medicine: supporting empathy and expertise in distributed systems. Educational Psychology Review, 10, 3-24. doi:10.1023/A:1022854014093.

Mayer, R. E. (2001). Multimedia learning. Cambridge: Cambridge University Press. 
Mayer, R. E., \& Moreno, R. (2003). Nine ways to reduce cognitive load in multimedia learning. Educational Psychologist, 38(1), 43-52. doi:10.1207/S15326985EP3801_6.

Mohammed, S., Klimoski, R., \& Rentsch, J. (2000). The measurement of team mental models: we have no shared schema. Organizational Research Methods, 3, 123-165. doi:10.1177/109442810032001.

Novak, J. D., \& Gowin, D. B. (1984). Learning how to learn (pp. 15-54). Cambridge: Cambridge University Press.

Paas, F., Renkl, A., \& Sweller, J. (2003). Cognitive load theory and instructional design: recent developments. Educational Psychologist, 38(1), 1-4. doi:10.1207/S15326985EP3801_1.

Schmidt, H. G., Norman, G. R., \& Boshuizen, H. P. A. (1990). A cognitive perspective on medical expertise: Theory and implications. Academic Medicine, 65, 611-621. doi:10.1097/00001888-199010000-00001.

Slavin, R. E. (1996). Research on cooperative learning and achievement: What we know and what we need to know. Contemporary Educational Psychology, 21, 43-69. doi:10.1006/ceps.1996.0004.

Visschers-Pleijers, A. J. S., Dolmans, D. H. J. M., de Grave, W. S., Wolfhagen, I. H. A. P., Jacobs, J. A., \& van der Vleuten, C. P. M. (2006). Student perceptions about the characteristics of an effective discussion during the reporting phase in problem-based learning. Medical Education, 40, 924-931. doi: 10.1111/j.1365-2929.2006.02548.x.

Westberg, J., \& Jason, H. (1994). Teaching creativity with video. New York: Springer Publishing Company. 